\title{
A Method for Measuring the Degree of Fermentation of the Edible Mushroom Cultivation Substrate
}

\author{
Jinping Zhang*, Xuebin Li, Yue Yin \\ Research Institute of Subtropical Forestry, Chinese Academy of Forestry, Hangzhou, China \\ Email: *jinpingzhang@126.com
}

How to cite this paper: Zhang, J.P., Li, X.B. and Yin, Y. (2018) A Method for Measuring the Degree of Fermentation of the Edible Mushroom Cultivation Substrate. Natural Resources, 9, 355-360. https://doi.org/10.4236/nr.2018.911022

Received: November 8, 2018

Accepted: November 27, 2018

Published: November 30, 2018

Copyright (c) 2018 by authors and Scientific Research Publishing Inc. This work is licensed under the Creative Commons Attribution International License (CC BY 4.0).

http://creativecommons.org/licenses/by/4.0/

\section{(c) (i) Open Access}

\begin{abstract}
In the study, eight treated substrates were designed to explore the possibility to determine the degree of fermentation of the substrate by the mycelial growth rate, whose main raw material includes composted pine sawdust, oil tea shell and hickory shell respectively, and auxiliary materials contain rice bran, soybean powder, etc. The result showed that the shiitake mushroom grew well in 7 treatments whose mycelial growth rate could be measured on $3^{\text {rd }}$ days when the mycelial growth rates of $\mathrm{P} 1, \mathrm{C} 1$ and $\mathrm{H} 1$ were $5.0 \mathrm{~mm} / \mathrm{d}$, $9.66 \mathrm{~m} / \mathrm{s}, 13.33 \mathrm{~m} / \mathrm{s}$. Auricularia cornea var. Li exhibited the fastest growth on P1 substrate. And mycelial growth rates of P1, P0 and CK1 were $5.8 \mathrm{~mm} / \mathrm{d}$, $3.66 \mathrm{~mm} / \mathrm{d}$, and $4.66 \mathrm{~mm} / \mathrm{d}$ on $3^{\text {rd }}$ day, respectively. The growth rates of Pleurotus geesteranus of C1, CK2 and P0 were $9.0 \mathrm{~mm} / \mathrm{d}, 11.66 \mathrm{~mm} / \mathrm{d}$, and $4.00 \mathrm{~mm} / \mathrm{d}$ on $3^{\text {rd }}$ day, respectively. So the degree of fermentation of the substrate could be determined within 3 days according to the mycelial growth rate. As the growth of edible fungi is affected by degree of fermentation of the substrate and there is little literature on degree of fermentation of edible fungi substrate, the study will provide theoretical and technical basis for determination of substrate fermentation.
\end{abstract}

\section{Keywords}

Mushroom Cultivation Substrate, Degree of Fermentation, Edible Fungi, Mycelium, Growth Rate

\section{Introduction}

The cultivation substrate of edible fungi provides the nutrients for their growth. Therefore, even under the same conditions, the composition, formula, and 
treatment of the substrate can significantly affect the mycelial growth rate, as well as the yield and flavor of the fruiting body [1]. A mushroom cultivation substrate is usually composed of a main component and auxiliary materials. Wood, herbs, feces, fiber, etc. with abundant carbons can be used as the main component of a mushroom cultivation substrate [1]. Organic nitrogen sources, phosphorus and potassium fertilizers, inorganic salts, etc. have been used as the auxiliary materials for the cultivation of mushrooms [1]. The mixed substrate can be used directly without any treatment, which makes the cultivation easy and time saving. However, the fermentation of the substrate can burn some mushroom spores, resulting in low germination rates, especially in summer when the substrate composts to acids [1]. Therefore, directly using raw substrates for mushroom cultivation requires larger amounts of spores. Another option is to use clinker substrates, which is usually applied to fungi with low decomposing abilities. In general, bagged substrates are sterilized under atmospheric pressure or autoclaved to kill harmful microorganisms, pests, and bacteria, to reduce contaminations, promote yield, and improve the stability of production [1].

The effects of the degree of fermentation of substrate on the mycelial growth rates of edible fungi have been reported by many researchers. For example, Xiao and Liu [2] cultivated Pleurotus cystidiosus using the defatted pine and fir sawdust, and evaluated the substrates with the mycelial growth rate. They found that the formulation of substrate significantly affected the mycelial growth rate and the bagged cultivation substrate was outgrown in $42-47$ days. Lin and Zhang [3] investigated the spawning and mycelial growth rates of edible fungi on the substrates containing different ratios of crushed cassava stems to sawdust. Zhang [4] treated pine sawdust by the lime water soaking method and stacking fermentation method as the cultivation substrate of fungi. They determined the degree of fermentation of the substrate with the aromatic scent. Tang [5] treated pine sawdust by the detoxification method and used the pine oil scent and alkaline odor as the indicators of the degree of fermentation. Qiu [6] evaluated the degree of fermentation of pine sawdust with the aromatic scent, color (yellow-brown), and feels (soft) during the natural composting.

In the study, substrates composed of pine sawdust, oil tea shell or hickory shell that were used as the main raw materials, and rice bran, soy flour, corn flour, and gypsum that were used as auxiliary materials with the moisture contents of 55\% - 60\% were inoculated with shiitake mushroom, Auricularia cornea var. Li and Pleurotus geesteranus spores, respectively, in test tubes. The mycelial length was used to evaluate the growth rate of mycelium and the degree of fermentation of the substrate. Our work has provided a simple and rapid method to determine the degree of fermentation of substrate, as well as a theoretical and technical foundation for studying the mycelial growth and substrate fermentation of edible fungi.

This study used the mycelial growth rates of edible fungi to determine the de- 
gree of substrate fermentation. According to some literatures at present, it is only aimed at the growth of edible fungi mycelium, and the degree of substrate fermentation is not determined. In the study, we can determine the degree of substrate fermentation by measuring the mycelial growth rates of edible fungi.

\section{Materials and Methods}

\subsection{Materials}

The shiitake mushroom, Auricularia cornea var. Li and Pleurotus geesteranus spores were provided by Wuhan SuisuiFeng Agricultural Technology Development Co., Ltd., (Hubei Province, China). The contents of terpenes in the untreated and composted Pinus massoniana sawdust were measured to be $0.396 \%$ and $0.096 \%$, respectively. The contents of saponin in the untreated and composted oil tea shell were $4.8 \%$ and $2.06 \%$, respectively.

\subsection{Experimental Design}

Eight substrates were prepared with different raw materials, the specific treatments were shown in Table 1. Each test was conducted in 4 replicates in the test tubes $\left(3 \mathrm{~cm} \mathrm{I.D.} \times 20.5 \mathrm{~cm} \mathrm{~L}\right.$ ). Each test tube contained $\sim 22 \mathrm{~cm}^{3}$ substrate that accounted $\sim 51 \%$ of the total volume of the test tube.

\subsection{Methods}

\subsubsection{Preparation of Substrate}

The components of each substrate were weighted and mixed, and the moisture content of the substrate was adjusted to $55 \%$ - $60 \%$ with water. The substrate was further mixed thoroughly, and filled in test tubes of the same size. Each tube was loaded with the same amount of substrate, and sterilized in an autoclave at $121^{\circ} \mathrm{C}$ for $20 \mathrm{~min}$.

\subsubsection{Inoculation}

The sterilized substrates in test tubes were cooled to room temperature on a

Table 1. Experimental design.

\begin{tabular}{ccc}
\hline Treat & Main raw material $(80 \%)$ & Auxiliary materials $(20 \%)$ \\
P0 & Untreated Pinus massoniana sawdust & \\
P1 0 & Composted Pinus massoniana sawdust & \\
C1 & Untreated oil tea shell & Rice bran 15\%+ soybean powder \\
H0 & Composted oil tea shell & powder $1 \%$ \\
H1 & Untreated hickory shell & \\
CK & Composted hickory shell & \\
CK1 & Weed tree sawdust & \\
CK2 & Weed tree sawdust & \\
\hline
\end{tabular}


clean bench, irradiated with a UV lamp for 30 minutes, inoculated with the solid edible fungus spores rapidly, and sealed.

\subsubsection{Cultivation and Measurement}

The inoculated substrates in tubes were cultured in an incubator at $24^{\circ} \mathrm{C}$ and the air humidity of $\sim 70 \%$. The lengths of mycelia were measured every 3 days to calculate the mycelial growth rate.

\section{Results and Discussion}

\subsection{Effects of Substrate on the Mycelial Growth Rate of Shiitake Mushroom}

As shown in Figure 1, all of the shiitake groups grew well, yet with different mycelial growth rates. The growth rates of the $\mathrm{P} 1, \mathrm{C} 1$ and $\mathrm{H} 1$ substrates were measured to be $1.66 \mathrm{~mm} / \mathrm{d}, 3.22 \mathrm{~mm} / \mathrm{d}$, and $4.44 \mathrm{~mm} / \mathrm{d}$, respectively, higher than those obtained on the $\mathrm{P} 0, \mathrm{C} 0$ and $\mathrm{H} 0$ substrates. The growth rate of $\mathrm{P} 1$ substrate was $5.0 \mathrm{mmd} / \mathrm{d}$ on the $3^{\text {rd }}$ day, and the mycelium is all over the tube on day 18 with the growth rate of $5.8 \mathrm{~mm} / \mathrm{d}$, indicating that the substrate was conducive to the growth of shiitake mushroom due to its suitable degree of looseness, high degree of fermentation, and low contents of tannins, saponins and terpenes. The mycelial growth rates on $\mathrm{C} 1$ and $\mathrm{H} 1$ substrates was $9.66 \mathrm{mmd} / \mathrm{d}$ and $13.33 \mathrm{mmd} / \mathrm{d}$, and the growth rate on day 18 were found to be $3.72 \mathrm{~mm} / \mathrm{d}$ and $5.78 \mathrm{~mm} / \mathrm{d}$, respectively, possibly due to the unsuitable matrix porosity that limited the access of the mycelia to oxygen. In addition, the substrates with low degrees of fermentation contained the substances that were in conducive to the growth of edible fungi and/or substances that inhibited the growth of edible fungus was produced during the fermentation. The $\mathrm{P} 0, \mathrm{C} 1, \mathrm{C} 0, \mathrm{H} 1, \mathrm{H} 0$, and $\mathrm{CK}$ substrates were outgrown with mycelia on day $22,40,43,26,32$, and 22 , respectively, suggesting that the composted pine sawdust, oil tea shell and hickory shell were more suitable for the germination and growth of edible fungi, than the corresponding raw materials.

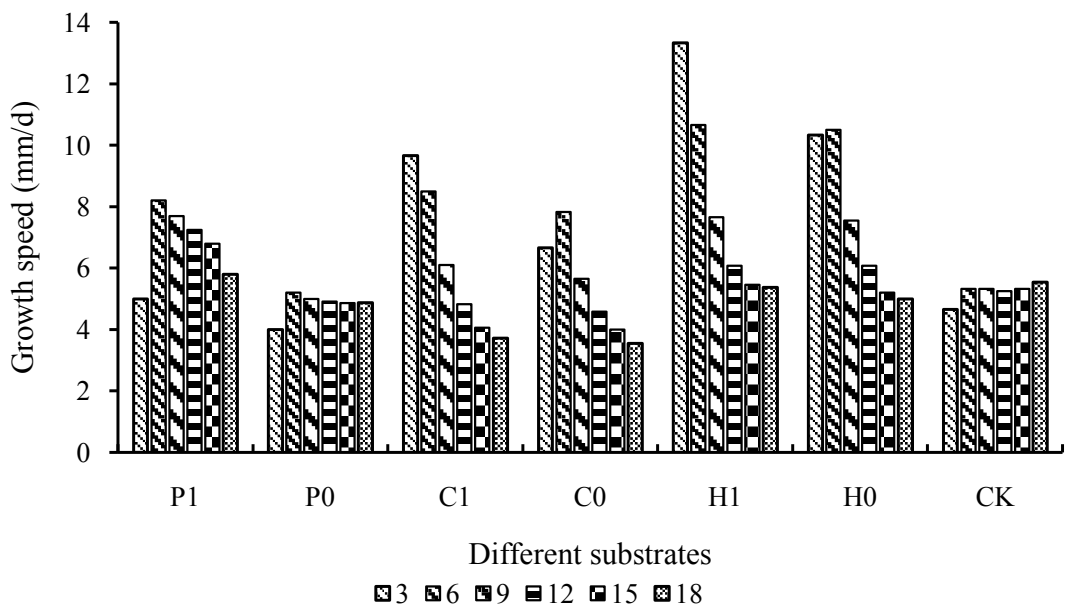

Figure 1. Mycelial growth rates of the seven shiitake mushroom groups (mm/d). 


\subsection{Growth of Auricularia cornea var. Li on Pinus massoniana Sawdust Substrates}

The Auricularia cornea var. Li mycelia could geminate and grow in the three treatment substrates with the growth rates in the order of P1 > CK1 > P0 (Figure 2). The daily mycelial growth rate using P1 substrate was measured to be 3.0 $\mathrm{mm} / \mathrm{d}$ which was similar to that of shiitake mushroom. And the growth rate on day 3 and day 18 was $6.0 \mathrm{~mm} / \mathrm{d}$ and $5.8 \mathrm{~mm} / \mathrm{d}$ respectively. The mycelium were all over the tubes with P0 and CK1 as substrates on day 27 and 22 with the growth rates of $3.66 \mathrm{~mm} / \mathrm{d}$ and $4.66 \mathrm{~mm} / \mathrm{d}$ on day 3 and the growth rate of 5.11 $\mathrm{mm} / \mathrm{d}$ on day 18. These results suggest that the content of terpenes in the Pinus massoniana sawdust, as well as its fermentation degree, promoted the growth of Auricularia cornea var. Li.

\subsection{Growth of Pleurotus Geesteranus on Oil Tea Shell Substrates}

Pleurotus geesteranus was geminated and grown only in $\mathrm{C} 1, \mathrm{C} 0$, and CK2 substrates (Figure 3), with the growth rates on day 18 of $3.55 \mathrm{~mm} / \mathrm{d}, 3.44 \mathrm{~mm} / \mathrm{d}$,

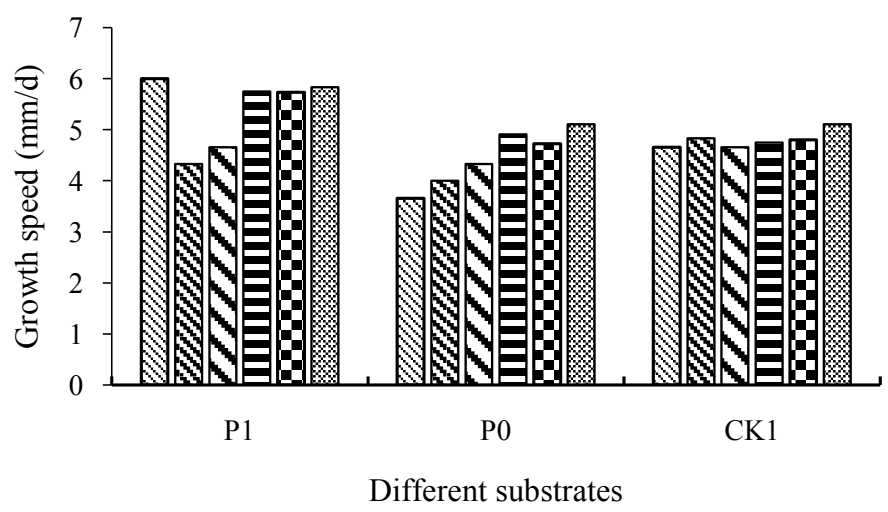

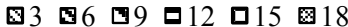

Figure 2. Growth rates of Auricularia cornea var. Li mycelia on P1, P0 and CK1 substrates $(\mathrm{mm} / \mathrm{d})$.

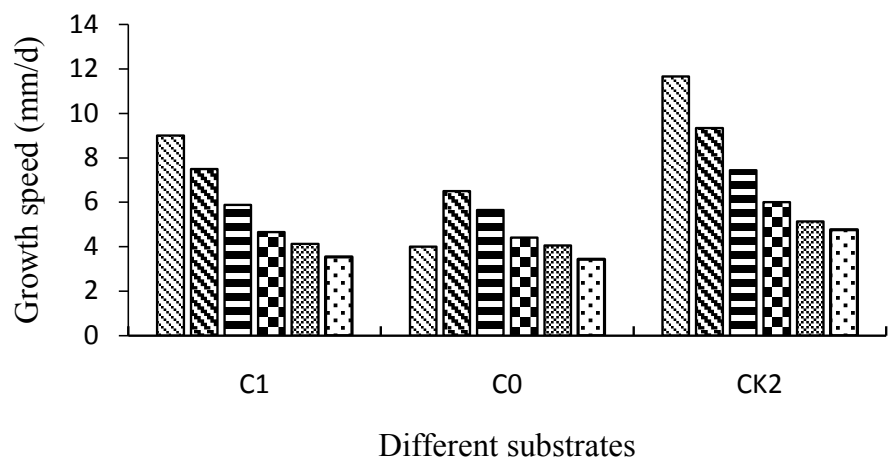

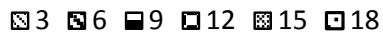

Figure 3. Growth rates of Pleurotus geesteranus mycelia on substrates $\mathrm{C} 1, \mathrm{C} 0$, and CK2 $(\mathrm{mm} / \mathrm{d})$. 
and $4.77 \mathrm{~mm} / \mathrm{d}$, respectively and the tubes of the three substrates were full of mycelium in 36, 42, and 27 days, respectively. The mycelial growth rate of Pleurotus geesteranus in C1 was $9.0 \mathrm{~mm} / \mathrm{d}$ on day 3 and $3.55 \mathrm{~mm} / \mathrm{d}$ on day 18 . And the growth rate in CK2 was $11.66 \mathrm{~mm} / \mathrm{d}$ on day 3 and $4.77 \mathrm{~mm} / \mathrm{d}$ on day 18 . The growth rate in $\mathrm{C} 0$ is slower than it in $\mathrm{C} 1$ and $\mathrm{CK} 2$, which was $4.0 \mathrm{~mm} / \mathrm{d}$ on day 3 and $3.44 \mathrm{~mm} / \mathrm{d}$ on day 18 . The low growth rate of $\mathrm{C} 1$ group was due to the low degree of looseness, as well as the compounds that inhibited the growth of edible fungi. The C0 group exhibited the slowest growth because of the harmful substances for the growth in the untreated oil tea shell, as well as the high content of hemicellulose [7] that caused the low porosity of the substrate.

\section{Conclusion}

In summary, the composted Pinus massoniana sawdust, oil tea shell and hickory shell are conducive to the germinations and growths of edible fungi. The mycelial growth rate can be used to determine the degree of fermentation of substrate in three days with appropriate amounts of substrate and liquid spawn. In addition, the degradation degree of substance in conducive to the growth of edible fungi can also be evaluated with the mycelial growth rate. The looseness and the content of hemicellulose of the substrate are the major factors affecting the growth of mycelia.

\section{Acknowledgements}

The authors are grateful for the financial support from National Key R \& D Program of China, Grant No. 2017YFD0601002 and the Provincial Department of Science and Technology of Zhejiang, China, Grant No. 2017C02022.

\section{Conflicts of Interest}

The authors declare no conflicts of interest regarding the publication of this paper.

\section{References}

[1] Dong, X.J. and Wang, X. (2008) Classification and Treatment of Edible Fungi Cultivation Substrates. Beijing Agriculture, No. 22, 27-28.

[2] Xiao, S.N., Liu, Y.G., Wong, C.F., et al. (2007) Abalone Mushroom Cultivation with Pine and Fir Sawdust. Edible Fungi, No. 2, 28.

[3] Lin, L.M., Zhang, Z.W., Cai, K., et al. (2017) Effects of Substrate on the Yields of Edible Fungi. Chinese Journal of Tropical Crops, 38, 2008-2013.

[4] Zhang, Z.W., Jiang, T., Wei, L.M., et al. (2006) Production of Edible Fungi with Fir Sawdust. Chinese Journal of New Agriculture, No. 5, 54.

[5] Tang, G.X. (1999) Detoxication of Fir Sawdust for the Cultivation of Edible Fungi. Chinese Journal of Edible Fungi, No. 3, 37.

[6] Qiu, G.G. (2004) Treatments of Pine and Fir Sawdust. Chinese Journal of Agricultural Technical Services, No. 4, 34.

[7] Zhang, J.P., Ying, Y., Li, X.B. and Yao, X.H. (2018) Evaluation of Three Kinds of Nutshell with Respect to Utilization as Culture Media. BioResources, 13, 7508-7518. 